\title{
Las redes sociales en la cultura digital: percepción, participación, movilización
}

\section{Social networks in digital culture: perception, participation and mobilisation}

María del Carmen García Galera, Universidad Rey Juan Carlos - carmen.garcia@urjc.es

Mercedes del Hoyo Hurtado, Universidad Rey Juan Carlos - mercedes.hoyo@urjc.es

Cristóbal Fernández Muñoz, Universidad Complutense de Madrid - cristobal.fernandez@ccinf.ucm.es

Resumen

Las redes sociales deben enfocarse cómo una forma de comunicación que tiene la capacidad de favorecer la comunicación entre los jóvenes y ayudar a su integración y participación en la vida social activa. Con este punto de partida, se puso en marcha en colaboración con la red social Tuenti, una investigación empírica cuyos objetivos se centran, en primer lugar, en conocer el grado de incidencia que tienen las redes sociales en la participación social activa de los jóvenes, es decir, cómo las redes sociales son la herramienta para la movilización social online/offline de los jóvenes; en segundo lugar, y dada la preocupación social al respecto, la investigación trata de analizar la percepción de privacidad/seguridad que los jóvenes tienen de redes sociales como Tuenti o Facebook.

Las repercusiones y el potencial tanto en positivo como en negativo que puede tener el uso de estas redes sociales lleva a plantear en el estudio empírico que aquí se presenta una primera hipótesis: la percepción de las redes como un ámbito seguro para la comunicación y la participación activa incrementa la propia participación de los jóvenes en las redes.

La observación de la realidad y los diferentes estudios sobre el tema ponen de manifiesto que hay que tener en cuenta otras variables que animan a los jóvenes a la movilización social activa como consecuencia de la participación en redes sociales. Así, la segunda hipótesis del estudio plantea que la implicación en actividades sociales y cívicas motivadas por las redes sociales depende también de factores como la cercanía o empatía del usuario con la situación. A los efectos de medir empíricamente las actitudes y percepciones de los jóvenes usuarios de redes sociales, se ha empleado la encuesta online que ha sido distribuida a través de la red social Tuenti.

\section{Palabras clave}

Redes sociales, jóvenes, seguridad, participación.

\section{Abstract}

Social networks should be approached as a form of communication that has the capacity to favour communication between young people and help their integration and participation in active social life. With this starting point, an empirical research was launched in collaboration with the social network Tuenti, whose objectives are focused, firstly, on knowing the degree of impact that social networks have on the active social participation of young people, i.e. how social networks are the tool for the online/offline social mobilisation of young people; secondly, and given the social concern in this regard, the research tries to analyse the perception of privacy/security that young people have of social networks such as Tuenti or Facebook.

The repercussions and the potential, both positive and negative, that the use of these social networks can have leads the empirical study presented here to propose a first hypothesis: the perception of the networks as a safe environment for communication and active participation increases young people's own participation in the networks. 


\section{Marco referencial}

Uno de los elementos que caracterizan la cultura digital en la que la sociedad desarrollada vive inmersa son las redes sociales. Entre la multitud de herramientas y aplicaciones que ofrece Internet, las redes sociales se han convertido en un elemento de referencia para la participación ciudadana de los jóvenes, ya que favorecen la posibilidad de ser informados y de informar de forma fácil, rápida y masiva, además de tener la capacidad de llamar a la movilización social y/o cívica. Las redes sociales se constituyen en realidad, como un espacio de encuentro, de debate, de movilización y, en definitiva, de participación. Como afirma Ben Rattray, activista digital y uno de los cien hombres más influyentes del mundo: "Nunca antes en la historia tuvo la gente tanto poder" (http://www.lavanguardia.com/lacontra/20121023/54353365305/la-contra-ben-rattray.htm)

Desde que aparecieron, redes sociales como Facebook, MySpace o Tuenti han acaparado la atención y el tiempo de millones de ciudadanos de todo el mundo. Mientras que la mayoría de estas nuevas formas de comunicación social centran su ámbito de actuación en el desarrollo y la consolidación de relaciones sociales ya existentes, hay otras cuya finalidad se centra en poner en contacto a personas que no se conocen entre sí pero que comparten unos mismos intereses, ideologías o actividades.

En este sentido, las redes sociales se pueden definir como un servicio web que permite a los individuos (1) construir un perfil público 0 semi-público dentro de un sistema limitado (2) articular una lista de otros usuarios con quien comparten conexión y (3) ver y explorar su lista de conexiones y aquellas hechas por otros usuarios dentro del propio sistema (Boyd y Ellison, 2007). Así pues, una red social es el resultado de la comunicación interpersonal y grupal que mantienen una serie de individuos a lo largo del tiempo. Se puede definir igualmente como un constructo analítico para comprender la dinámica social y una relación entre personas que consideran a otros miembros de la red como importantes o relevantes para ellos de alguna forma (Welman, 1996). Otro concepto de interés en este contexto es el de las web de redes sociales, las páginas de Internet que permiten construir un perfil público o semipúblico y articular relaciones de manera que suele ser visible para todos aquellos que acceden a su perfil (García, Alonso y del Hoyo, 2013)

De hecho, más del $90 \%$ de los datos disponibles online en la actualidad se han generado en los dos últimos años, en gran medida por la proliferación de redes sociales, que han permitido hacer más sencilla la comunicación en tiempo real.

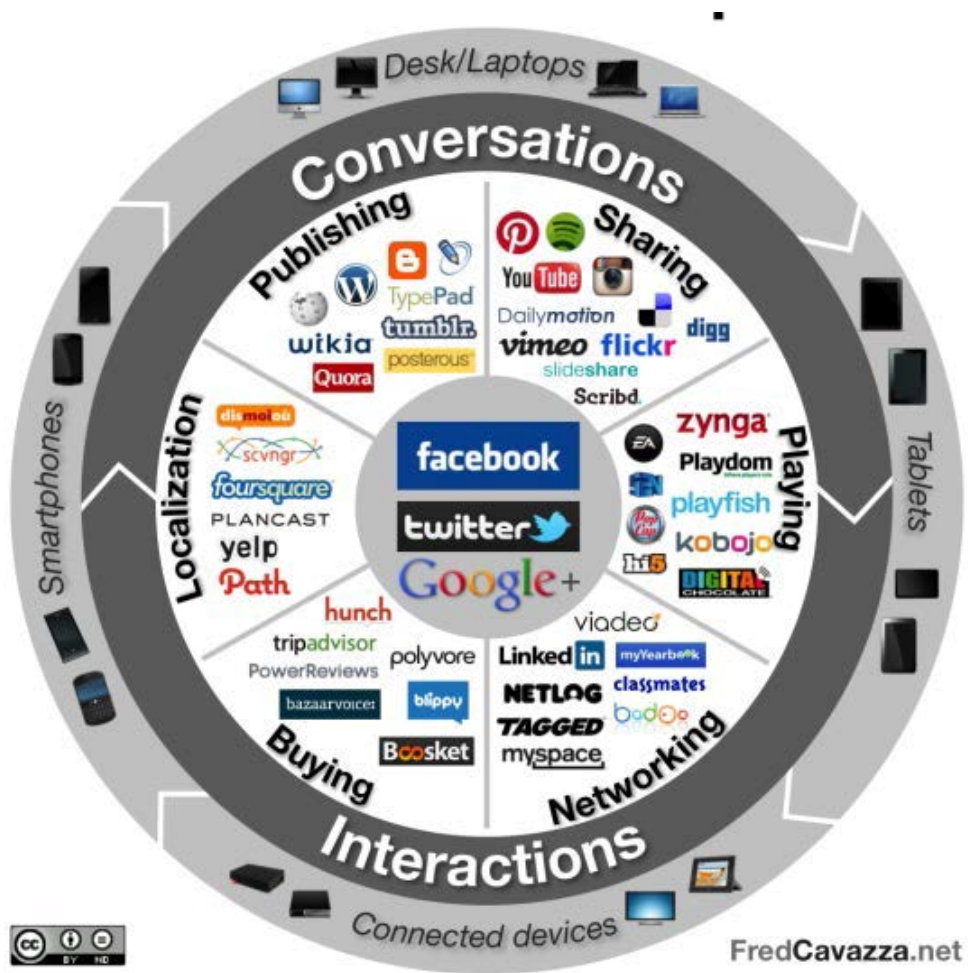

El planteamiento de que las redes sociales son un elemento exclusivamente de riesgo y asociado a usos ilícitos o indebidos es una visión muy parcial de una realidad que presenta muchas más caras. Es necesario cambiar esta percepción de las redes y presentar también cómo esta forma de comunicación tiene la capacidad de ser una herramienta que favorece la comunicación entre los jóvenes y que ayudan a su integración y participación en la vida social activa.

Si bien los estudios sobre los usos de Internet en general y de las redes sociales en particular se han multiplicado en los últimos tiempos ${ }^{1}$, en su inmensa mayoría llegan a verificar una realidad observada por casi todos y es que los jóvenes, como principales

\footnotetext{
${ }^{1}$ El artículo de Boyd y Ellison (2007) hace una importante recopilación de las principales investigaciones realizadas en Estados Unidos sobre los usos de las redes sociales.
} 
usuarios de las redes sociales, las utilizan fundamentalmente para prolongar sus relaciones sociales con su grupo de iguales a través del mundo virtual (Ellison, Steinfield y Lampe, 2007; García y Del Hoyo, 2013). Desde este medio de comunicación, los usuarios se convierten en emisores de contenidos, que comparten abiertamente con sus amigos y, en algunas ocasiones, también con desconocidos. No obstante, los datos reflejan que el porcentaje de jóvenes que utilizan las redes para contactar con personas a las que no conoce no es significativo cuantitativamente, si bien hay que tener presente que las consecuencias de un uso indebido de las redes con desconocidos sí puede tener cualitativamente unos efectos negativos que habría que abordar desde otras áreas de estudio (Livingstone, Haddon y Görzig, 2012)

En este sentido, los datos muestran que apenas un 10\% de los jóvenes utilizan las redes para establecer nuevas amistades. El estudio realizado por Muscanell y Guadagno (2012) entre 238 jóvenes universitarios, ofrece algún dato más al respecto y refleja que son los hombres jóvenes los que utilizan las redes sociales con más frecuencia para hacer nuevas amistades, mientras que las mujeres declararon que utilizaban las redes para mantener las relaciones de amistad que ya tenían con anterioridad,

No obstante, el objetivo de esta comunicación no se centra en las relaciones sociales que se crean o refuerzan a través de las redes online. En esta comunicación, la cuestión que se plantea se orienta hacia otro propósito: por un lado, qué límites se pueden o deben poner al uso de esta forma de comunicación social digital y, por otro, qué otras formas de participación -más allá de las relaciones sociales- tienen los jóvenes en las redes sociales online. No es incompatible que los usuarios de las redes las utilicen como forma de comunicación entre su grupo de iguales y que vean otra funcionalidad -y de hecho, la apliquen- a las redes sociales. Las horas que les dedican son muchas y, además, la tecnología móvil hace posible que las barreras tradicionales de tiempo y espacio hayan casi desaparecido. El usuario puede acceder a las redes sociales en cualquier momento y desde cualquier dispositivo; es más, el futuro ya se prevé con un teléfono móvil como principal tecnología que nos permitirá responder a todas nuestras necesidades cotidianas de convivencia: desde acceder a las redes sociales -como es ya una realidad- a miles de actos cotidianos a los que la telefonía móvil dará respuesta.

Pew Research Global Actitude Project, en un estudio realizado al respecto en 2012, presenta un ranking con los países cuyos ciudadanos utilizan el teléfono móvil para conectarse a las redes sociales. En primer lugar se sitúa Israel (53\%) y le sigue de cerca Estados Unidos (50\%). España se sitúa en quinta posición, con un $42 \%$ de ciudadanos que utilizan el teléfono móvil para acceder a las redes (http://www.pewglobal.org/2011/12/20/global-digital-communication-texting- social-networking-popular-worldwide/ -Consultado el 9 de octubre de 2013)

Adicionalmente, para los jóvenes, utilizar Internet significa principalmente acceder a las redes sociales. En uno de los estudios realizados recientemente por el Centro de Investigaciones Sociológicas sobre jóvenes y el uso de las nuevas tecnologías, se preguntaba a este sector de la población para qué usaban principalmente Internet. Un porcentaje muy importante respondió que para "buscar información y documentación" (82\%) y, en segundo lugar, el 79,6\% afirmaba que para "acceder a las redes sociales" http://www.cis.es/cis/export/sites/default/archivos/Marginales/2880_2899/2889/ES2889.pdf Consultado el 9 de octubre de 2013).

Las herramientas online de comunicación social han revolucionado las oportunidades de comunicación de los individuos. En muy poco tiempo su uso se ha generalizado. Sus funcionalidades han multiplicado las posibilidades de interacción, el grado de inmediatez de las mismas y su alcance. Se ha llegado a hablar de la nueva democracia de la comunicación gracias al impacto de las herramientas sociales.

Así las cosas, parece necesario avanzar en el campo de la difusión científica dando a conocer cómo los jóvenes participan en acciones sociales colectivas online y offline y cómo actúan frente a las cuestiones de privacidad y seguridad en la red. Por ello, se desarrolló una encuesta online que contaría con la colaboración de Tuenti para alcanzar a la muestra deseada. Concretamente, se ha desarrollado como herramienta de investigación un cuestionario online adaptado a las condiciones propias de las redes sociales. La encuesta a través de Internet tiene unas características intrínsecas, como son la rapidez en la recogida de información, el bajo coste y/o la mejora en las respuestas que se adaptaban al estudio que aquí se realiza (Díaz de Rada, 2012). Los sitios web que albergan redes sociales ofrecen ahora ciertas ventajas para realizar encuestas a gran escala. En esta ocasión, para la recogida de datos, se contó con la colaboración de Tuenti, la red social española por excelencia para los adolescentes y jóvenes que puso en marcha una campaña publicitaria en su plataforma -con 10 millones de usuarios activos- para divulgar y animar a la respuesta del cuestionario entre sus usuarios (el $80 \%$ de la actividad en Tuenti corresponde a usuarios entre 14 y 25 años). Desde dicha campaña en Tuenti, se incluía un enlace al cuestionario de la investigación referido a su participación en general en redes sociales, no exclusivamente en Tuenti. La muestra seleccionada para el estudio está formada por 1.330 personas, jóvenes de España, mediante muestreo probabilístico aleatorio simple, con un intervalo de confianza del $95,5 \%$ y $p=q=50 \%$ y un margen de error es de más/menos $2,7 \%$. A continuación, se exponen y revisan los resultados obtenidos.

\section{Seguridad en las redes}

Uno de los primeros estudios sobre la privacidad y seguridad en las redes sociales, lo encontramos en Gross y Acquisti (2005), quienes analizaron a 4000 estudiantes universitarios y señalaron en ese momento las amenazas potenciales a la privacidad contenidas en la información personal que los estudiantes incluían en sus perfiles. Estos autores argumentaban que, con frecuencia, había una cierta contradicción entre el deseo de los jóvenes de proteger su privacidad y sus conductas reales. Es lo que Barnes (2006) describió como la "paradoja de la privacidad", que tiene lugar cuando los adolescentes no son conscientes de la naturaleza pública de Internet, y publicitan en sus perfiles información que afecta a su vida privada y que, probablemente, no compartirían en la vida real (Utz y Kramer, 2009). 
Cada día se presentan ejemplos del uso desafortunado de las redes sociales. Famosos con comentarios inadecuados en sus perfiles, fotos o vídeos no deseados difundidos masivamente, actitudes arrogantes de perfiles corporativos frente a los usuarios 0 desgraciadamente, casos de delitos cometidos usando las redes sociales como instrumento. Ocurre que en ocasiones, la estimación del riesgo -alta o baja- por parte de los individuos es la variable que marca la decisión personal de incluir datos o información personal en las redes (Akcora, Carminati y Ferrari, 2012)

Uno de los primeros pasos que sugieren las voces expertas para evitar problemas asociados a la publicidad de esos datos personales es que los usuarios de las redes se lean las condiciones de privacidad antes de darse de alta en cualquier red social. Los datos obtenidos en el estudio realizado entre más de 1300 adolescentes y jóvenes usuarios de redes sociales reflejan que el 51\% no se leyó las condiciones de privacidad y seguridad cuando se dieron de alta en Facebook o en Twitter. No obstante, los usuarios de Tuenti sí parecen estar más preocupados por este aspecto de las redes y el 72,3\% manifiesta haberse leído las indicaciones que ofrece esta red social a sus usuarios antes de darse de alta. Es más, al 95,2\% le gusta el sistema de privacidad de Tuenti porque solo sus amigos pueden ver su perfil y su tablón, si bien el $41 \%$ de los jóvenes encuestados afirman que les gustaría tener algunas opciones más de privacidad.

Otro dato interesante en este sentido se encuentra en la información personal que los jóvenes comparten en su perfil en las redes. Hay un $90 \%$ que manifiesta que publica su nombre real y su edad en las redes sociales, y un $99 \%$ su sexo. Sin embargo, en cuestiones como la situación sentimental, un $60 \%$ manifiesta que la refleja en su perfil, un $40 \%$ comparte su número de teléfono y un $85 \%$ informa al resto de sus amigos en las redes sobre el centro en el que estudia.

Recordemos que aunque en ocasiones pueda parecer lo contrario, los datos personales no pertenecen sino a los usuarios, no a las redes sociales y plataformas en las que los usuarios participan. Algunas redes sociales parecen haberlo olvidado cuando hacen imposible borrar un perfil o limitar el acceso a la información privada con métodos complejos de gestión. Por lo tanto, al contrario de lo que ocurre en las Vegas, lo que ocurre en las redes sociales, no siempre permanece en las redes sociales. No obstante, algunas redes sociales como Tuenti han hecho precisamente de la privacidad un elemento diferencial y facilitan la comunicación entre usuarios en su ámbito personal de manera totalmente confiable.

Frente al contexto general de internet, en Tuenti existe un marco de actuación muy claro y comprometido a este aspecto, hasta el punto de poder afirmar que es una de las redes sociales más seguras y la que cuenta con la política de privacidad más estricta, tal y como señalan distintas organizaciones sociales que operan en este ámbito. Así, por ejemplo, la organización Protégeles ha afirmado que Tuenti es la red social más privada y segura de las que operan en España (http://www.seguridadweb20.es/tuenti.php, consultado 25 de noviembre de 2013). De hecho sólo permite la participación de personas reales y no indexa los perfiles de sus usuarios en resultados de buscadores, lo que supone una gran diferencia con otras redes sociales. Por defecto, todos los perfiles tienen activada las máximas opciones de privacidad de forma que la información personal, las fotos, los contenidos privados solo las vean los amigos, previniendo la recepción de mensajes de desconocidos o evitando la descarga de fotos. Se trata de funcionalidades muy seguras y el $100 \%$ de los usuarios de Tuenti tiene activado el máximo nivel de protección de su privacidad al registrarse.

Todas las redes sociales deberían cumplir escrupulosamente con la ley e incluso ir más allá estableciendo mecanismos de control adicionales, con el filtrado de páginas para menores de edad o la verificación de la edad mínima de los usuarios, que cualquier usuario pueda reportar perfiles y fotos inadecuadas, o una respuesta rápida a las consultas de seguridad y privacidad. Desde Tuenti declaran que en los últimos dos años las incidencias de privacidad se han reducido un 52\% como consecuencia de la mayor sensibilización y las mejoras introducidas. Sin embargo, y según el mismo informe de la asociación "Protégeles", entre las redes sociales que dan consejos sobre seguridad e información destinada específicamente a los adolescentes, de forma que además sea fácil de encontrar y de entender no se encuentra Facebook.

Desde diferentes ámbitos se incide en la necesidad de andar con seguridad por las redes online y proponen, entre otras medidas, por ejemplo, que las redes sociales cuenten y pongan a disposición de los usuarios un centro de ayuda y seguridad; publicitar con vídeos y recomendaciones de buen uso de la red como no aceptar como amigos a personas que no conozcan previamente; realizar periódicamente acciones de sensibilización con padres y educadores. Las redes sociales se han asentado de manera tan rápida en la vida de todos los individuos que se necesitan ciertos medios, ciertas herramientas que faciliten su conocimiento y uso adecuado. Las nuevas tecnologías entran en la vida de las personas, jóvenes y mayores, y hay que adoptarlas y adaptarlas a la cotidianeidad. Asumir las normas básicas de la convivencia que se comparten en la vida real a la dimensión online propiciará una participación en las redes sociales más seguras y enfocadas hacia otras perspectivas, como las de la participación en acciones sociales colectivas, de las que pasamos a hablar a continuación.

\section{Participación social activa desde las redes sociales}

España se sitúa como el quinto país del mundo que más utiliza las redes sociales, principalmente, Tuenti, Facebook y Twitter. Así, por ejemplo, Tuenti cuenta con 10 millones de usuarios activos y 15 millones de usuarios registrados (http://corporate.tuenti.com/es/blog/10- millones-de-usuarios-activos-15-millones-de-usuarios-registrados-muchas-gracias, consultado 25 de noviembre de 2013) Facebook tiene 18 millones de usuarios activos mensuales en España, de los cuales 12 millones acceden todos los días. En cuanto al acceso en dispositivos móviles tienen 13 millones de accesos mensuales de los cuales 8,1 millones de personas acceden todos los días (http://alt1040.com/2013/09/facebook-espana consultado 25 de noviembre de 2013). Twitter ya alcanza los 5,6 millones y Linkedln los 2,7 millones en 2013, lo que supone un crecimiento de más de un millón de usuarios en estas dos redes respecto a 2012 (http://www.concepto05.com/2013/07/estadisticas-usuarios-redes-sociales-en-espana-2013/, consultado 25 de 
Los primeros resultados descriptivos de la realidad de los usos de estas redes por parte de este sector de la población y obtenidos de la encuesta realizada entre más de 1300 jóvenes, ponen de manifiesto que la red social que cuenta con una mayor presencia de jóvenes es Tuenti, de hecho, el $89 \%$ de los encuestados tienen perfil en esta red social y el $70 \%$ declaran que sus amigos también lo están. Les sigue Facebook, con un $85 \%$ y Twitter, con un $60 \%$ de usuarios. En relación al tiempo que los jóvenes invierten dentro de estas redes sociales, el 61,3\% de los encuestados afirma utilizar Tuenti varias veces al día, el $45 \%$ afirma tener esta misma rutina en Facebook y el $40 \%$, en Twitter. No obstante, cualquiera que sea la red social que empleen, el $80 \%$ de los jóvenes declara que las redes sociales forman parte de su vida diaria y difícilmente podrían pasar un día sin acceder a ellas.

Facebook y otras redes sociales están cambiando la forma en que nos enfrentamos a nuestras acciones cotidianas diariamente: cómo compramos, cómo nos relacionamos o cómo estamos informados. Adicionalmente, percibir que se "circula" con seguridad por las redes anima a la participación en las mismas.

La inmensa mayoría de los adolescentes y jóvenes cibernautas están presentes en las redes con una actitud positiva, bien como receptores de información o bien como emisores de contenido que les ayuda a relacionarse con su grupo de iguales, a estar informados o a compartir información. Así, el $21 \%$ de jovenes encuestados manifestaron que utilizan las redes sociales para recomendar libros, películas de cine, e incluso, algún museo. Interesante también resulta el porcentaje de jóvenes que responden que utilizan las redes sociales como medio para informarse sobre hechos y/o acontecimientos sociales y/o políticos. Así, hay un $64,2 \%$ que afirmar utilizar las redes con este fin, y un $51 \%$ declara que utiliza las redes para animar a otros a la participación en algunas jornadas, manifestaciones 0 convocatorias.

Así pues, las redes sociales no se deben encasillar en un medio únicamente utilizado por los adolescentes y jóvenes para extender las relaciones sociales. La realidad demuestra que las redes son también un medio para la información y para mostrar una actitud cívica constructiva, es decir, una herramienta para la participación en la vida social activa.

Organizaciones del Tercer Sector como Amnistía Internacional, Greenpeace o Change.org ya son conscientes de la importancia que tienen las redes sociales para animar a la participación social activa de los ciudadanos, y de hecho, redes sociales como Facebook se han convertido en una herramienta clave para organizar y coordinar protestas de carácter cívico en muchísimas ciudades alrededor del mundo. Con las redes sociales, el poder de convocatoria se ha visto ampliado y el coste de la realización de campañas de concienciación social ha mermado considerablemente. Y es que desde estos soportes se captan la atención de más usuarios que con las campañas de captación a pie de calle. La razón es obvia: pulsar un botón para unirse a un grupo es gratis. Estamos hablando de las que podríamos denominar "Causas 2.0", situaciones que demandan la solidaridad y la cooperación de los ciudadanos y que utilizan las redes sociales para su movilización.

¿Qué tienen las redes sociales para influir en sus usuarios que no hayan tenido con anterioridad otros medios de comunicación, como la televisión, cuyos efectos en las audiencias -y movilización de las mismas a través del medio- se han estudiado durante décadas? La respuesta enlaza dos rasgos obvios: la inmediatez y la interactividad. En este nuevo entorno, los usuarios no desempeñan básicamente un único papel de receptores (papel que apenas habían abandonado en el proceso de comunicación que establecían los mass media tradicionales), sino que asumen alternativamente tanto el papel de receptores como el de emisores, alternancia casi innata a la comunicación interpersonal que ahora, gracias al desarrollo tecnológico, se traslada a la comunicación global.

Estos nuevos canales han cambiado los parámetros de la comunicación entre individuos y colectivos, permitiendo que el diálogo se democratice y multiplique exponencialmente. Se trata, en definitiva, de una nueva actitud y un nuevo marco de posibilidades para la comunicación social y el activismo.

Los jóvenes son importantes generadores de contenidos en las redes, y muy activos. Sus principales usuarios y sus principales protagonistas. De hecho, en los resultados obtenidos de la encuesta realizada entre los más de 1300 jóvenes usuarios de las redes, se muestra que el $84 \%$ de los jóvenes entrevistados declaran generar con asiduidad comentarios en las redes; el 47,5\% colabora aportando opiniones sobre noticias que aparecen en redes y el $40,4 \%$ genera información sobre eventos con esa misma frecuencia. No obstante, el porcentaje se incrementa exponencialmente cuando se trata de compartir material más personal como pueden ser fotografías, en el que el $87,4 \%$ lo hace y el $53,8 \%$, que comparte vídeos.

Ahora bien. En relación a la participación de los jóvenes en eventos que requieren de su colaboración, se produce entre este grupo dos percepciones encontradas. En primer lugar, quienes consideran importante la existencia de este tipo de eventos, por la finalidad que encierran de implicación, colaboración, participación y movilización social colectiva de las personas pertenecientes a la red, para alcanzar un objetivo de interés general. En este sentido, puede ser significativo el $18 \%$ de jóvenes que manifiestan haberse inscrito a alguna ONG tras haber sido animado a ello desde las propias redes sociales o el $21 \%$ de jóvenes que declara haber participado en movilizaciones de la Universidad en el propio campus porque ha sido informado a través de las redes sociales.

En segundo lugar, se encuentra aquella posición de quienes desconfían de la buena fe y de los objetivos altruistas de determinados eventos, y que consideran que obedecen más a intereses particulares encaminados a subir o elevar el número de visitas en un perfil determinado. En estos casos, el ruido por la proliferación de convocatorias consideradas superfluas y distantes organizadas para seducir a los potenciales clientes, afecta a la credibilidad de la información recibida (García, Alonso y del Hoyo, 2013) 
Los jóvenes del primer grupo, es decir, aquellos que no solamente consideran importante que haya invitaciones en las redes a estos eventos, sino que participan en los mismos, expresan su participación de dos formas diferentes. Una primera es la que les anima a participar simplemente con un acto online, es decir, con el click del ratón manifiestan un "me gusta", que contrariamente a lo que algunos pudieran pensar, es un acto cargado de significado para organizaciones como Greenpeace, Change.org y otras entidades de gran calado social pertenecientes al Tercer Sector.

Una segunda es la que les motiva a traspasar la actuación online y llevarla a la vida real. Las variables que inciden en adoptar una u otra conducta por parte de los jóvenes parecen encontrarse, entre otras, en la proximidad geográfica con el evento en sí, si bien aún son más los que se movilizan sin moverse del mundo virtual que aquellos que optan por expresar su solidaridad o cooperación en el mundo real. Las cifras, por ejemplo, cuando se trata de participar en una "campaña en defensa de la escuela del barrio" reflejan que hay un $31 \%$ de jóvenes que acudiría a alguna movilización, si bien el 40\% afirma que participaría con un "click". No obstante, y para demostrar la afirmación de la proximidad geográfica, solo un $17 \%$ contestó que acudiría a una movilización contra la pena de muerte en Irán, y el $44,5 \%$ lo haría a través de las redes sociales. Es el predominio del "click-activismo" entre la generación de los nativos digitales.

Pero ese click-activismo está teniendo un gran significado en un gran número de movilizaciones que se están desarrollando desde muy diferentes ámbitos, y es que su significado no se reduce a apoyar exclusivamente la promoción de una causa o una campaña a través de las redes sociales. Es el uso de los medios digitales para facilitar el cambio social y el activismo, tomando como protagonistas a los jóvenes y a las redes sociales que lo facilitan (http://www.clicktivist.org/what-is-clicktivism/ consultado 26 de noviembre de 2013).

\section{Conclusiones}

La privacidad en la "plaza pública" que son las redes sociales ha venido ganando importancia a medida que se ha extendido la toma de conciencia sobre las implicaciones de la participación en las mismas. Su uso, el de las redes, se ha extendido antes que el conocimiento de la utilización adecuada de las mismas y, en no pocas ocasiones, el aprendizaje ha llegado a través del método ensayo-error, de traspiés, accidentes y fracasos.

Mantener la privacidad de los datos e información que se expone en las redes sociales online se convierte en un reto, en ocasiones, ya que incluso con el usuario más preocupado por su privacidad, encuentra dificultades ante las propias políticas de privacidad de las redes, que cambian constantemente. Sin embargo, redes sociales como Tuenti han conseguido equilibrar la participación de jóvenes y adolescentes en sus entornos virtuales y la seguridad de la navegación en el mundo de la comunicación social digital.

Por otra parte, y asociado a la seguridad y privacidad en las redes, en tanto que sentirse más seguro en cuanto a la información que se expone en la red y se comparte ayuda a una mayor participación en las mismas, el estudio realizado entre los jóvenes españoles a través de la plataforma de Tuenti ha demostrado que los jóvenes utilizan las redes sociales para participar en acciones de solidaridad y cooperación.

Movimientos sociales, alimentados lógicamente con la participación más o menos activa de muchos individuos, han existido siempre, pero las nuevas tecnologías digitales y las nuevas dimensiones que con ellas alcanza el concepto de interacción, dan a sus usuarios mayor poder en relación con dichos movimientos, desde el instante en que los convierten en emisores de contenido con que contribuir a la movilización, en colaboradores activos necesarios como individuos para conseguir el objetivo colectivo.

La participación en actos de solidaridad o cooperación puede adoptar diferentes formas, que suponen una mayor o menor implicación online u offline. En este sentido, los jóvenes han expresado su actitud de solidaridad, en tanto que casi un $80 \%$ de los encuestados han manifestado su apoyo a las campañas a las que se les podría invitar a participar, todas ellas con diferentes perspectivas geográficas, sociales o emocionales. Sin embargo, su implicación activa en esas campañas varía, predominando los que colaboran sin salir de las redes sociales sobre los que acudirían a una movilización en la vida real por defender una causa. Como siguiente paso, sería necesario y de gran interés conocer qué variables inciden en la movilización de estos jóvenes, online u offline, y qué determina su participación social activa.

\section{Referencias bibliográficas}

Akcora, Cuneit G., Carminati, Barbara, Ferrari, Elena. (2012) Privacy in Social Networks: How Risky is Your Social Graph?. Data Engineering (ICDE), 2012 IEEE 28th International Conference. DOI:10.1109/ICDE.2012.99

Andrews, Liu. (2011) I Know who you are and I saw what you did. Free Press, Nueva York

Barnes, Susan (2006). A privacy paradox: Social networking in the United States. First Monday, Vol. 11, num. 9 http://firstmonday.org/htbin/cgiwrap/bin/ojs...

Boyd, Danah M. y Ellison, Nicolle B. (2008) Social Network Sites: Definition, History, and Scholarship. Journal of ComputerMediated Communication, num. 13, pp. 210-230 
Díaz de Rada, Vidal (2012). "Ventajas e inconvenientes de la encuesta por internet", Papers- Revista de Sociología (ISSN 0210-2862), n 97/1, pp. 193-223.

Ellison, Nicolle B., Steinfield, Charles y Lampe, Cliff. (2007). The benefits of Facebook "friends": Exploring the relationship between college students' use of online social networks and social capital. Journal of Computer-Mediated Communication, 12(3)

García Galera, María del Carmen y Del Hoyo Hurtado, Mercedes (2013). Redes sociales. Un medio para la movilización juvenil. Revista Zer, vol. 18, num. 34, pp.111-125

García Galera, María del Carmen, Alonso Seco, José y Del Hoyo Hurtado, Mercedes (2013). La participación de los jóvenes en las redes sociales: finalidad, oportunidades y gratificaciones. Revista Analisi, pp.95-110

Muscanell, Nicole L. y Guadagno Rosanna E.(2012) Make new friends or keep the old: Gender and personality differences in social networking use. Computers in Human Behavior 28(1): 107-112

Livingstone, Sonia, Haddon Leslie y Görzig, Anke (2012). Children, Risk and Safety on the Internet. Research and Policy challenges in comparative perspective. University of Bristol, Gran Bretaña.

Utz, Sonja y Kramer, Nicolle. (2009). The privacy paradox on social network sites revisited: The role of individual characteristics and group norms. Cyberpsychology: Journal of Psychosocial Research on Cyberspace, 3(2), article 1.

Welman, Barry (1996). Computer Networks as Social Networks: Collaborative Work, Telework, and Virtual Community Annual Review of Sociology, Vol. 22: pp. 213-238 DOl:

10.1146/annurev.soc.22.1.21 